\title{
Produção de serapilheira e retorno de nutrientes em um trecho de Mata Atlântica secundária, na Reserva Biológica de Paranapiacaba, SP1
}

\author{
MARISA DOMINGOS², REGINA M. DE MORAES ${ }^{2}$, YARA S. DE VUONO² e \\ CARLOS E. ANSELMO ${ }^{2}$
}

(recebido em 02/01/95; aceito em 04/12/96)

\begin{abstract}
Litter production and nutrient return in a portion of the secondary Atlantic Forest, at the Biological Reserve of Paranapiacaba, SP). The litter production and nutrient contents have been quantified in an area of Atlantic Forest at the Biological Reserve of Paranapiacaba, in the state of São Paulo, free from the impact of the air pollution from the industrial complex of Cubatão. The objectives of this study were to compare the results obtained here with those of a similar study performed at more and less affected areas of the Reserve and to determine the extension of the air pollution impacts at that region. Thirty litter trays with $0,25 \mathrm{~m}^{2}$ were randomly distributed, sampling the litter monthly during one year (Abr/88 to Mar/89). The samples were sorted by leaves, twigs, reproductive parts and miscellaneous, weighed and $\mathrm{N}, \mathrm{P}, \mathrm{K}, \mathrm{Ca}, \mathrm{Mg}$ and $\mathrm{S}$ concentrations were determined. The annual total litter production was $7007 \mathrm{~kg} \cdot \mathrm{ha}^{-1}$ ( $72 \%$ of leaves, $22 \%$ of twigs, $4 \%$ of miscellaneous and $2 \%$ of reproductive parts) The litterfall and nutrient fluxes were continuous along the year, without any clear seasonal pattern. Leaves showed higher levels of $\mathrm{Ca}$ and $\mathrm{Mg}$ while miscellaneous presented more N, P, K and S. The macronutrient return through total litterfall followed the decreasing sequency $\mathrm{N}>\mathrm{Ca}>\mathrm{S}>\mathrm{K}>\mathrm{Mg}>\mathrm{P}$. Through comparison with results from other studies on tropical forests and on the Paranapiacaba Reserve itself, it is concluded that the part of the forest here studied does not present changes caused by air pollution in this step of nutrient cycling.
\end{abstract}

RESUMO - (Produção de serapilheira e retorno de nutrientes em um trecho de Mata Atlântica secundária, na Reserva Biológica de Paranapiacaba, SP). A produção e o conteúdo de nutrientes da serapilheira foram quantificados em área de Mata Atlântica na Reserva Biológica de Paranapiacaba, estado de São Paulo, não afetada pela poluição aérea do pólo industrial de Cubatão. Os objetivos do estudo foram realizar uma comparação com resultados de trabalho semelhante, desenvolvido em áreas da Reserva mais e menos afetadas pela poluição e determinar a extensão dos impactos desse estresse naquela região. Utilizaram-se 30 coletores de $0,25 \mathrm{~m}^{2}$ com coletas mensais durante um ano (abr/88 a mar/89). As amostras mensais foram separadas em folhas, ramos, partes reprodutivas e miscelânea, pesadas e analisadas quanto aos teores de N, P, K, Ca, Mg e S. A produção anual de serapilheira total foi de $7007 \mathrm{~kg}^{-h^{-1}}(72 \%$ de folhas, $22 \%$ de ramos, $4 \%$ de miscelânea e $2 \%$ de partes reprodutivas). A produção de serapilheira foi contínua ao longo do ano, sem apresentar efeitos de sazonalidade. As folhas apresentaram os maiores teores de $\mathrm{Ca}$ e $\mathrm{Mg}$, e a miscelânea, os maiores de $\mathrm{N}, \mathrm{P}, \mathrm{K}$ e $\mathrm{S}$. O retorno de macronutrientes pela serapilheira total produzida obedeceu à sequiência decrescente dada por $\mathrm{N}>\mathrm{Ca}>\mathrm{S}>\mathrm{K}>\mathrm{Mg}>\mathrm{P}$. Com base em comparações com resultados obtidos em outros estudos desenvolvidos em florestas tropicais e na própria Reserva de Paranapiacaba, conclui-se que o trecho da floresta estudado não apresenta alterações causadas pela poluição nessa etapa da ciclagem de nutrientes.

Key words - Litterfall, nutrient return, Atlantic Forest

\section{Introdução}

O estudo da produção de serapilheira e do fluxo de nutrientes minerais associado é de fundamental importância para a compreensão dos aspectos dinâmicos dos ecossistemas. Sua quantificação tem sido realizada em trabalhos desenvolvidos em diversas partes do mundo (Proctor 1984) e também no Brasil, sendo que nas regiões de Mata Atlântica preservada existem os estudos de Jackson (1978), em Nova Lombardia (ES); Delitti (1987), na Juréia (SP); Varjabedian \& Pagano (1988), no Guarujá (SP); Oliveira \& Lacerda (1993), no Rio de Janeiro (RJ) e Moraes (1993), na Ilha do Cardoso (SP).

1. Desenvolvido dentro do Programa Linhas de Ação em Botânica do CNPq.

2. Seção de Ecologia, Instituto de Botânica, Caixa Postal 4005, 01061-970 São Paulo, SP, Brasil.
Em menor número, porém, são os trabalhos realizados com o objetivo de detectar influências de atividades antrópicas sobre essa etapa da ciclagem mineral, como os de Domingos et al. (1990), em áreas da Reserva de Paranapiacaba mais expostas à poluição oriunda do pólo industrial de Cubatão, e de Leitão Filho (1993), também em regiões da Serra do Mar, nas proximidades daquele pólo.

Parte dos poluentes emitidos em Cubatão é transportada pelos ventos através do vale do rio Mogi atingindo a Reserva de Paranapiacaba, que se situa no alto da Serra do Mar. Trechos da floresta mais próximos ao vale mostram sinais de degradação mais evidentes do que outros mais afastados.

Projeto realizado naquele local, em que vários aspectos da ciclagem de nutrientes foram abordados comparativamente em duas áreas da Reserva, localizadas nas proximidades da porção mais alta do vale do rio Mogi e distintas quanto ao grau de 
perturbação da vegetação, indicou que houve indícios de alterações na produção, decomposição e estoque de serapilheira, mesmo na parte da floresta considerada fisionomicamente menos afetada pela poluição (De Vuono et al. 1989, Domingos et al. 1990, Lopes et al. 1990).

Partindo-se da hipótese de que locais mais afastados do vale do rio Mogi não seriam afetados pela poluição aérea, decidiu-se realizar este estudo da produção mensal e anual de serapilheira e do fluxo de nutrientes minerais associado, em área no interior da Reserva, supostamente livre da ação dos poluentes, para confrontar seus resultados com os obtidos em Domingos et al. (1990) e, através dessa abordagem comparativa entre valores observados em porções da floresta perturbadas e não perturbadas, verificar o impacto desse estresse nos processos ecológicos da floresta.

\section{Material e métodos}

Área de estudo - A Reserva Biológica de Paranapiacaba localiza-se a $23^{\circ} 46^{\prime} \mathrm{S}$ e $48^{\circ} 18^{\prime} \mathrm{W}$, município de Santo André, estado de São Paulo. Possui área de 336 ha, relevo tipicamente montanhoso, com altitudes entre 750 e 890 m. Apresenta clima do tipo super-úmido, segundo a classificação de Thornthwaite, ou $\mathrm{Cfb}$, de acordo com o sistema de Köppen, precipitação média de $3380 \mathrm{~mm}$ anuais e temperatura média anual de $18^{\circ} \mathrm{C}$ (Funari et al 1986). O tipo de solo dominante é o latossolo vermelho amarelo. A vegetação é classificada como floresta latifoliada perenifolia ou floresta pluvial tropical de encosta, conhecida genericamente como Mata Atlântica. A área de amostragem utilizada neste estudo situa-se próxima à zona de transição entre Mata Atlântica e campo natural. É coberta por mata secundária, com grande ocorrência de indivíduos pertencentes à família Melastomataceae, de acordo com levantamento fitossociológico.

Metodologia - Para o estudo da produção de serapilheira foram utilizados 30 coletores com superfície de $0,25 \mathrm{~m}^{2}$, construídos com sarrafos de madeira e tela de náilon de $1 \mathrm{~mm}$ de abertura e apoiados a $15 \mathrm{~cm}$ acima do solo. Os coletores foram instalados em 10 parcelas de $5 \times 10 \mathrm{~m}$, escolhidas ao acaso entre aquelas demarcadas para o levantamento fitossociológico, sendo colocados três em cada uma, que constituíram repetições por parcela. As coletas foram mensais, durante o período de um ano, de abril/1988 a março/1989. O material recolhido em cada coletor, após secagem prévia ao ar, foi separado nas frações folhas, ramos, partes reprodutivas e miscelânea (materiais não identificados), que permaneceram em estufa, a $70^{\circ} \mathrm{C}$, até atingirem peso constante. Após pesagem, as três repetições de cada parcela foram reunidas por fração, obtendo-se assim, 10 amostras de serapilheira separadas em quatro frações, as quais foram moídas em moinho de lâminas. As frações folhas e ramos foram moídas separadamente por amostra. As frações partes reprodutivas e miscelânea, devido à pequena quantidade de material, foram reunidas e, então, moídas, obtendo-se uma amostra mista de partes reprodutivas e duas amostras mistas de miscelânea. Alíquotas dessas amostras foram, mensalmente, submetidas a digestões sulfúrica e nítrico-perclórica para determinação de seus conteúdos de N, P, K, Ca, Mg e S, segundo Zagatto et al. (1981), realizada pelo Centro de Energia Nuclear na Agricultura (CENA), em Piracicaba. Os resultados de peso seco foram expressos em kg.ha-1 e quanto às concentrações, em $\%$ de peso seco. A produção de serapilheira em cada mês foi comparada estatisticamente através dos testes F e Tukey.

\section{Resultados e Discussão}

Produção de serapilheira - A produção de serapilheira ocorreu ao longo de todo o período de observação apresentando, entretanto, considerável variação entre os meses (figura 1). O mês de maior produção foi setembro (847 kg.ha $\left.{ }^{-1}\right)$ e o de menor, julho (337 kg.ha-1 $)$, portanto com uma variação entre o valor máximo e o mínimo da ordem de 2,5 vezes, diferença significativa ao nível de 5\% (teste Tukey).

O total anual estimado foi de $7007 \mathrm{~kg} \cdot \mathrm{ha}^{-1}$, valor que se encontra em posição intermediária ao verificado em outras florestas tropicais (Proctor 1984), incluindo a Mata Atlântica (tabela 1), situando-se na faixa prevista por Bray \& Gorham (1964) para a latitude em que a floresta se encontra. Considerando-se apenas a fração folhas, cuja amostragem é mais uniforme, verifica-se maior semelhança do resultado aqui obtido $\left(5081 \mathrm{~kg} \cdot \mathrm{ha}^{-1}\right)$ aos relatados em outras áreas cobertas por Mata Atlântica (tabela 1). Contudo, o total anual de serapilheira produzida difere muito do verificado

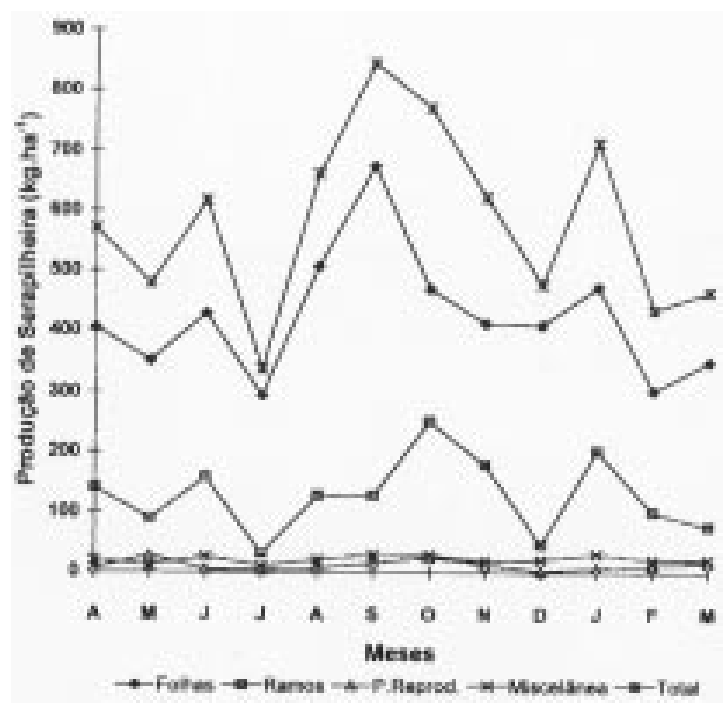

Figura 1. Produção de serapilheira e de suas frações, em kg.ha-1, na Reserva Biológica de Paranapiacaba, SP. 
Tabela 1. Produção de serapilheira em áreas cobertas por Mata Atlântica e na região sudeste do Brasil (t.ha ${ }^{-1}$.ano $\left.{ }^{-1}\right)$.

\begin{tabular}{lccl}
\hline Local & Serapilheira total & Fração folhas & \multicolumn{1}{c}{ Referência } \\
\hline Rio de Janeiro & 8,9 & 6,5 & Oliveira \& Lacerda 1993 \\
Rio de Janeiro & 9,0 & 6,0 & Louzada et al.1995 \\
Espírito Santo & - & 4,6 & Jackson 1978 \\
São Paulo & 7,9 & 5,0 & Varjabedian \& Pagano 1988 \\
São Paulo & 6,3 & 4,4 & Moraes 1993 \\
São Paulo & 5,1 & 4,1 & Leitão Filho 1993 \\
São Paulo (mais afetada) & 1,8 & - & Domingos et al. 1990 \\
São Paulo (menos afetada) & 3,8 & - & Domingos et al. 1990 \\
São Paulo & 7,0 & 5,1 & Este estudo \\
\hline
\end{tabular}

Tabela 2. Concentrações médias anuais de macronutrientes e respectivos desvios padrões (\%PS) nas frações da serapilheira produzida na Reserva Biológica de Paranapiacaba, SP.

\begin{tabular}{ccccc}
\hline Elementos & Folhas & Ramos & Part. reprod. & Miscelânea \\
\hline N & $2,40 \pm 0,20$ & $1,74 \pm 0,19$ & $2,42 \pm 0,64$ & $3,03 \pm 0,41$ \\
$\mathrm{P}$ & $0,10 \pm 0,01$ & $0,07 \pm 0,02$ & $0,12 \pm 0,05$ & $0,15 \pm 0,04$ \\
$\mathrm{~K}$ & $0,18 \pm 0,04$ & $0,10 \pm 0,03$ & $0,23 \pm 0,07$ & $0,23 \pm 0,05$ \\
$\mathrm{Ca}$ & $1,39 \pm 0,07$ & $1,00 \pm 0,14$ & $0,90 \pm 0,33$ & $0,98 \pm 0,22$ \\
$\mathrm{Mg}$ & $0,17 \pm 0,03$ & $0,07 \pm 0,02$ & $0,15 \pm 0,04$ & $0,14 \pm 0,02$ \\
$\mathrm{~S}$ & $0,21 \pm 0,03$ & $0,16 \pm 0,03$ & $0,17 \pm 0,04$ & $0,27 \pm 0,15$ \\
\hline
\end{tabular}

por Domingos et al. (1990), em estudo prévio realizado em outros trechos desta Reserva, em que se registraram $1803 \mathrm{~kg} \cdot \mathrm{ha}^{-1}$.ano ${ }^{-1}$ na área considerada mais afetada pela poluição e $3810 \mathrm{~kg} \cdot \mathrm{ha}^{-1} \cdot$ ano $^{-1}$, na menos afetada.

Segundo Johnson et al. (1982), a produção de serapilheira não é diretamente afetada pela poluição atmosférica, especialmente com referência à chuva ácida, mas influenciada pela simplificação da estrutura da vegetação (Smith 1981) e mudanças nos fluxos de elementos minerais (Mayer 1983) causadas pelo estresse. Esse fato não foi observado na porção da floresta estudada, por encontrar-se mais no interior da Reserva, a cerca de $2 \mathrm{~km}$ das utilizadas por Domingos et al. (1990), em local mais protegido dos ventos provenientes de Cubatão, resultando em um índice de produção semelhante ao obtido em outras florestas não afetadas por poluição aérea.

Variações nas condições climáticas entre os anos estudados podem ter contribuído para as diferenças verificadas entre os resultados aqui obtidos e aqueles de Domingos et al. (1990), mas, embora não existam registros meteorológicos feitos durante este último levantamento, sabe-se que não houve ocorrência de eventos como geadas ou secas, que poderiam provocar maior produção de serapilheira.

A relação entre a produção de serapilheira e a distribuição das chuvas (média de 23 anos, Funari et al. 1986) não foi nítida, fato já observado por alguns autores como Varjabedian \& Pagano (1988), Pagano (1989a) e Oliveira \& Lacerda (1993), embora em regiões onde não ocorra estação seca, como Paranapiacaba, vários estudos indiquem uma tendência à maior produção no verão, quando as condições ambientais são mais favoráveis à renovação das folhas (Delitti 1987, Leitão-Filho 1993, Moraes 1993), fato observado também por Domingos et al. (1990).

$\mathrm{Na}$ distribuição percentual das frações da serapilheira, as folhas tiveram a maior participação (72\%), valor bastante próximo ao estimado por O’Neill \& De Angelis (1980) para florestas tropicais (71\%), seguidas por ramos (22\%), miscelânea (4\%) e partes reprodutivas (2\%), sendo este último valor consideravelmente baixo. Leitão-Filho (1993), trabalhando em área sob intensa poluição, verificou que apenas $0,33 \%$ da serapilheira produzida correspondia a partes reprodutivas. Concluíram que 
a vegetação da área estudada caminhava para seu declínio, com as espécies canalizando a maior parte de sua energia na manutenção vegetativa. A porcentagem de partes reprodutivas aqui verificada, embora baixa, não possibilita conclusão semelhante, principalmente quando confrontada com a quantidade total de serapilheira produzida.

Conteúdo e transferência de nutrientes - A variação das concentrações dos elementos ao longo dos meses foi pequena e não demonstrou tendência sazonal nítida. Os teores foram próximos aos de Domingos et al. (1990) em seu sítio experimental menos perturbado, mas são superiores aos verificados em Mata Atlântica preservada por Moraes (1993).

A fração miscelânea apresentou os teores mais elevados de N, P, Ke S (tabela 2), possivelmente por ser constituída por materiais provenientes de todas as outras frações e material de origem animal, principalmente excrementos e fragmentos de artrópodes.

As concentrações de $\mathrm{Ca}$ e $\mathrm{Mg}$ foram mais elevadas na fração folhas. Os tecidos lenhosos da fração ramos apresentaram os menores teores de todos os macronutrientes, exceto de $\mathrm{Ca}$, que é um elemento estrutural.

A fração partes reprodutivas mostrou variações grandes em termos de concentração, ao longo dos meses e em comparação com as demais, devido a sua composição variável: flores, frutos e sementes de diversas espécies caindo em diferentes épocas do ano.

O retorno mensal de elementos minerais ao solo, via serapilheira, acompanhou mais proximamente a

Tabela 3. Estimativa da quantidade anual $\left(\mathrm{kg} \cdot \mathrm{ha}^{-1} \cdot \mathrm{ano}^{-1}\right)$ de elementos minerais transferidos para a superfície do solo através da serapilheira na Reserva Biológica de Paranapiacaba, SP.

\begin{tabular}{crrcrr}
\hline Elementos & Folhas & Ramos & Part. reprod. & Miscelânea & Total \\
\hline $\mathrm{N}$ & 120,76 & 26,72 & 3,41 & 7,82 & 158,71 \\
$\mathrm{P}$ & 5,11 & 1,08 & 0,62 & 0,14 & 6,95 \\
$\mathrm{~K}$ & 9,51 & 1,34 & 0,27 & 0,53 & 11,65 \\
$\mathrm{Ca}$ & 70,76 & 15,50 & 1,22 & 2,43 & 89,91 \\
$\mathrm{Mg}$ & 8,51 & 2,00 & 0,17 & 0,35 & 11,03 \\
$\mathrm{~S}$ & 10,65 & 2,11 & 0,19 & 0,53 & 13,48 \\
\hline
\end{tabular}

Tabela 4. Transferência de macronutrientes para o solo através da serapilheira em algumas florestas tropicais $\left(\mathrm{kg} \cdot \mathrm{ha}^{-1} \cdot \mathrm{ano}^{-1}\right)$.

\begin{tabular}{lrrrrrrrl}
\hline Tipo de flotresta (localização) & N & P & K & Ca & Mg & S & IEN* & Referência \\
\hline Terra firme (Amazonas-Br) & 115 & 4 & 29 & 115 & 16 & - & 70 & Dantas \& Phillipson 1989 \\
Terra firme (Roraima-Br) & 118 & 7 & 49 & 64 & 24 & - & 79 & Scott et al. 1992 \\
Terra firme (Pará-Br) & 156 & 4 & 17 & 33 & 27 & - & 63 & Klinge 1977 \\
Pluvial montana (Costa Rica) & 110 & 5 & 17 & 72 & 15 & - & 81 & Heaney \& Proctor 1989 \\
Pluvial montana (Venezuela) & 69 & 4 & 33 & 43 & 14 & - & 101 & Fassbender \& Grimm 1981 \\
Mesófila (São Paulo-Br) & 189 & 9 & 39 & 104 & 18 & - & 50 & Meguro et al. 1979 \\
Mesófila (São Paulo-Br) & 198 & 7 & 48 & 149 & 27 & 15 & 43 & Pagano 1989b \\
Mesófila (São Paulo-Br) & 203 & 6 & 43 & 109 & 24 & 14 & 44 & César 1993a, b \\
Atlântica (Rio de Janeiro-Br) & - & - & 55 & 77 & 27 & - & - & Oliveira \& Lacerda 1993 \\
Atlântica (São Paulo-Br) & 102 & 4 & 21 & 60 & 19 & 15 & 62 & Moraes 1993 \\
Atlântica (Cubatão/vale do Pilões) & 128 & 3 & 14 & 56 & 13 & 13 & 44 & Leitão Filho 1993 \\
Atlântica (Cubatão/vale do Pilões) & 98 & 2 & 11 & 30 & 9 & 10 & 45 & Leitão Filho 1993 \\
Atlântica (Cubatão/vale do Mogi) & 135 & 7 & 18 & 80 & 7 & 21 & 39 & Leitão Filho 1993 \\
Atlântica (São Paulo/mais afetada) & 46 & 2 & 6 & 22 & 3 & 7 & 39 & Domingos et al. 1990 \\
Atlântica (São Paulo/menos afetada) & 82 & 3 & 10 & 38 & 5 & 13 & 46 & Domingos et al. 1990 \\
Atlântica (São Paulo-Br) & 159 & 7 & 12 & 90 & 11 & 13 & 44 & Este estudo \\
\hline
\end{tabular}

* Índice de eficiência de uso de nutrientes, estimado a partir do retorno de N via queda serapilheira (Vitousek 1982). 
curva de produção de serapilheira do que a referente às concentrações, o que confirma a proposição de Delitti (1989) sobre a relevância da massa de folhedo produzido na determinação da magnitude dos fluxos de nutrientes.

A tabela 3 apresenta as quantidades anuais de nutrientes minerais na serapilheira total e em suas frações e pode-se notar que a maior parte dos nutrientes foi transferida ao solo pelas folhas, visto constituírem a fração preponderante na composição da serapilheira e apresentarem concentrações elevadas de quase todos os elementos analisados. $\mathrm{O}$ retorno de macronutrientes na serapilheira total obedeceu à seqüência decrescente dada por $\mathrm{N}>\mathrm{Ca}>\mathrm{S}>\mathrm{K}>\mathrm{Mg}>\mathrm{P}$.

Os resultados obtidos situam-se dentro da faixa verificada em florestas tropicais (tabela 4), o que sugere não haver interferência da poluição no fluxo de elementos na área. Com exceção dos valores de $\mathrm{K}$ e $\mathrm{Mg}$, que podem ser considerados baixos, $\mathrm{N}, \mathrm{P}$, $\mathrm{Ca}$ e $\mathrm{S}$ apresentaram-se em quantidades semelhantes à média, aproximando-se dos encontrados em algumas matas mesófilas semidecíduas pelas quais, freqüentemente, há uma grande circulação de nutrientes (Pagano 1989b). Em área preservada na Ilha do Cardoso, Moraes (1993), estimou um retorno anual de $\mathrm{N}, \mathrm{P}$ e $\mathrm{Ca}$ inferior ao aqui relatado. No estudo de Domingos et al. (1990), a transferência de nutrientes estimada também foi bastante inferior, até mesmo na área considerada menos afetada, mas isto foi devido principalmente à pequena quantidade de serapilheira produzida.

A grande variação entre os valores apresentados na tabela 4 decorre de diferenças na quantidade e na qualidade da serapilheira, na metodologia empregada, bem como de condições edafo-climáticas (Meguro et al. 1979, Fassbender \& Grimm 1981, Jordan \& Herrera 1981). Ela confirma a observação de Delitti (1995) de que a diversidade das florestas tropicais se manifesta também em seus padrões funcionais.

A relação entre a quantidade de serapilheira produzida e a quantidade de nutrientes minerais nela contida indica a eficiência de cada comunidade na utilização desses nutrientes (Vitousek 1982, 1984). Bridgham et al. (1995) aprimoram os preceitos de Vitousek $(1982,1984)$ e apresentam um novo modelo para avaliação da eficiência de uso dos nutrientes, onde é previsto que a produção de serapilheira aumenta assintoticamente com o aumento do retorno de $\mathrm{N}$ e $\mathrm{P}$ através desta e que o índice de eficiência, calculado de acordo com Vitousek (1982), diminui em função do aumento do retorno desses nutrientes via serapilheira. No presente estudo, obteve-se valor (tabela 4) semelhante aos índices estimados para algumas matas mesófilas paulistas (Meguro et al. 1979, Pagano 1989b, César 1993a, b) e para um trecho de Mata Atlântica não afetado pela poluição aérea, no vale do rio Pilões em Cubatão. Esse valor pode ser considerado baixo, mas é compatível com o retorno relativatnente alto do nutriente via serapilheira, segundo o modelo proposto por Bridgham et al. (1995). Os índices verificados no estudo de Domingos et al. (1990) foram semelhantes ao aqui apresentado, porém com uma produção de serapilheira bem inferior, estando abaixo do previsto no referido modelo.

Comparando-se os resultados obtidos nos dois estudos, pode-se concluir que, na porção da Reserva Biológica de Paranapiacaba estudada, a produção de serapilheira e retorno de nutrientes não se encontram alterados pela poluição atmosférica, como foi observado nas outras duas áreas mais próximas ao vale do rio Mogi.

\section{Referências bibliográficas}

BRAY, J.R. \& GORHAM, E. 1964. Litter production in forests of the world. Adv. Ecol. Res. 2:101-139.

BRIDGHAM, S.D., PASTOR, J., McCLAUGHERTY \& RICHARDSON, C.J. 1995. Nutrient-use efficiency: a litterfall index, a model, and a test along a nutrient-availability gradient in North Carolina peatlands. Am. Nat. 145:1-21.

CÉSAR, O. 1993a. Produção de serapilheira na mata mesófila semidecídua da Fazenda Barreiro Rico, município de Anhembi, SP. Revta brasil. Biol. 53:671-681.

CÉSAR, O. 1993b. Nutrientes minerais na serapilheira produzida na mata mesófila semidecídua da Fazenda Barreiro Rico, município de Anhembi, SP. Revta brasil. Biol. 53:659-669.

DANTAS, M. \& PHILLIPSON, J. 1989. Litterfall and litter nutrient content in primary and secondary amazonian terra-firme forest. J. Trop. Ecol. 5:27-36.

DE VUONO, Y.S., DOMINGOS, M. \& LOPES, M.I.M.S. 1989. Decomposição da serapilheira e liberação de nutrientes na floresta da Reserva Biológica de Paranapiacaba, sujeita aos poluentes atmosféricos de Cubatão, SP. Hoehnea 16:179-193.

DELITTI, W.B.C. 1987. Produção de folhedo na mata pluvial tropical da Estação Ecológica da Juréia, SP. Ciên. Cult. 39(Supl):600-601.

DELITTI, W.B.C. 1989. Ciclagem de nutrientes minerais em matas ciliares. Anais do Simpósio sobre Mata Ciliar, p.88-98. 
DELITTI, W.B.C. 1995. Estudos da ciclagem de nutrientes: instrumentos para análise funcional de ecossistemas terrestres. Oecologia Brasiliensis 1:469-486.

DOMINGOS, M., POGGIANI, F., DE VUONO, Y.S. \& LOPES, M.I.M.S. 1990. Produção de serapilheira na floresta da Reserva Biológica de Paranapiacaba, sujeita aos poluentes atmosféricos de Cubatão, SP. Hoehnea 17:47-58.

FASSBENDER, H.W \& GRIMM, V. 1981. Ciclos biogeoquímicos en un ecosistema florestal de los Andes Ocidentales de Venezuela. II. Producción y descomposición de los residuos vegetales. Turrialba 31:39-47

FUNARI, F.L., DE VUONO, Y.S. \& SALUM, S.T. 1986. Balanço hídrico de duas áreas de Mata Atlântica: Reserva Biológica de Paranapiacaba e Parque Estadual da Ilha do Cardoso (São Paulo). In Anais do IV Congresso da SBSP, p.95-101.

HEANEY, A. \& PROCTOR, J. 1989. Chemical elements in litter in forests on Vólcan Barva, Costa Rica. In Mineral nutrients in tropical forest and savanna ecosystems (J. Proctor, ed). Blackell Sci. Publi., Oxford, p.255-271.

JACKSON, J.F. 1978. Seasonality of flowering and leaf fall in a brazilian subtropical lower montane moist forest. Biotropica 10:38-42.

JOHNSON, D.W., TURNER, J. \& KELLY, J.M. 1982. The effects of acid rain on forest nutrient status. Wat. Resources Res. 18:449-461.

JORDAN, C.F. \& HERRERA, R. 1981. Tropical rain forests: are nutrients really critical? Am. Natur. 117:167-180.

KLINGE, H. 1977. Fine litter production and nutrient return to the soil in three natural forest stands of Eastern Amazonia. Geo-Eco-Trop 1:159-167.

LEITÃO-FILHO, H. de F. (coord.) 1993. Ecologia da Mata Atlântica em Cubatão. EDUNESP/EDUNICAMP, São Paulo.

LOPES, M.I.M.S., DE VUONO, Y.S. \& DOMINGOS, M. 1990. Serapilheira acumulada na floresta da Reserva Biológica de Paranapiacaba, sujeita aos poluentes atmosféricos de Cubatão, SP. Hoehnea 17:59-70.

LOUZADA, M.A.P., QUINTELA, M.F.S. \& PENNA, L.P.S 1995. Estudo comparativo da produção de serrapilheira em áreas de Mata Atlântica: a floresta secundária “antiga” e uma floresta secundária (capoeira). Oecologia Brasiliensis $1: 61-74$

MAYER, R. 1983. Interaction of forest canopies with atmospheric constituints: aluminium and heavy metals. In Effects of accumulation of air pollutants in forest ecosystems (B. Ulrich \& J. Pankrath, eds.). D. Reidel, Dordrecht, p.47-55.
MEGURO, M., VINUEZA, G.N. \& DELITTI, W.B.C. 1979. Ciclagem de nutrientes minerais na mata mesófila secundária - São Paulo. I. Produção e conteúdo de nutrientes minerais no folhedo. Bolm. Bot. Univ. S. Paulo 7:11-31.

MORAES, R.M. 1993. Ciclagem de nutrientes minerais em Mata Atlântica de encosta e mata sobre restinga, na Ilha do Cardoso, Cananéia, SP: produção de serapilheira e transferência de nutrientes. Dissertação de mestrado, Universidade de São Paulo, São Paulo.

OLIVEIRA, R. \& LACERDA, L.D. 1993. Produção e composição química da serapilheira na Floresta da Tijuca (RJ). Revta. brasil. Bot. 16:93-99.

O’NEILL, R.V. \& DE ANGELIS, D.L. 1980. Comparative productivity and biomass relations of forest ecosystems. In Dynamic properties of forest ecosystems (D. Reichle, ed.) Cambridge University Press, Carmbridge, p.411-449.

PAGANO, S.N. 1989a. Produção de folhedo em mata mesófila semidecídua no município de Rio Claro, SP. Revta brasil. Biol. 49:633-639.

PAGANO, S.N. 1989b. Nutrientes minerais do folhedo produzido em mata mesófila semidecídua no município de Rio Claro, SP. Revta brasil. Biol. 49:641-647.

PROCTOR, J. 1984. Tropical forest litterfall. II. The data set. In Tropical rain forest: the Leeds Symposium (S.L. Sutton \& A.C. Chadwick, eds.). Leeds Phil. Lit. Soc., Leeds, p.83-113.

SMITH, W.H. 1981. Air pollution and forest interactions between air contaminants and forest ecosystem. Springer-Verlag. New York.

SCOTT, D.A., PROCTOR, J. \& THOMPSON, J. 1992. Ecological studies on a lowland evergreen rain forest on Maracá Island, Roraima, Brazil. II. Litter and nutrient cycling. J. Ecol. 80:705-717

VARJABEDIAN, R. \& PAGANO, S.N. 1988. Produção e decomposição de folhedo em um trecho de Mata Atlântica de encosta no município do Guarujá, SP. Acta Bot. Bras. 1:243-256.

VITOUSEK, P.M. 1982. Nutrient cycling and nutrient use efficiency. Am. Nat. 119:553-572.

VITOUSEK, P.M. 1984. Litterfall, nutrient cycling, and nutrient limitation in tropical forests. Ecology 65:285-298.

ZAGATTO, E.A.G., JACINTHO, A.O., REIS, B.F., KRUG, F. J., BERGAMMIN-FILHO, H., PESSENDA, L.C.R., MORTATTI, J. \& GINÉ, M.F. 1981. Manual de análise de plantas e águas empregando sistemas de injeção em fluxo CENA. Piracicaba. 